\title{
ON THE EXISTENCE OF $L_{\infty x}$-INDISCERNIBLES
}

\author{
P. C. EKLOF
}

ABstract. It is proved that it $T$ is a countable theory of $L_{\omega_{1} \omega}$ with enough axioms for Skolem functions and with arbitrarily large models, then for any order type, there is a model of $T$ with a set of $L_{\infty x}$-indiscernibles of that order type.

In this short note we answer in the affirmative a question of Chang [4] as to whether there exist $L_{k x}$-indiscernibles of any given order type. In fact we prove a somewhat stronger result since we show the existence of $L_{\infty x}$-indiscernibles and we use a stronger definition of indiscernibles. Our result also gives a simpler proof of Chang's theorem on the existence of $L_{\kappa \kappa}$-indiscernibles of any well-ordered order type [4, Theorem 4]. (We thank Jon Barwise for some helpful discussions.)

In general we follow the notation of Chang [4] (so $\kappa$ is always an infinite regular cardinal). Let $L$ be a first order language with countably many relation, function, and constant symbols, and let $\mathfrak{U}=\langle A, \cdots\rangle$ be a structure for $L$. An ordered subset $X$ of $A$ is said to be $L_{\lambda \kappa}$-indiscernible if for any subset $Y$ of $X$ of cardinality $<\kappa$ and any order-preserving injection $h: Y \rightarrow X$,

$$
(\mathfrak{I}, y)_{y \in Y} \equiv \lambda_{\kappa}(\mathfrak{I}, h y)_{y \in Y} .
$$

If $L_{\infty x}$ is the union of the infinitary languages $L_{\lambda x}$, where $\lambda$ ranges over all cardinals, we define $L_{\infty}$-indiscernibles analogously.

Let $T$ be a countable theory of $L_{\omega_{1} \omega}$. There is a countable fragment $\mathfrak{L}_{A}$ such that $T \subseteq \mathfrak{L}_{A}$ (for the definition of $\mathfrak{L}_{A}$ see Barwise [1]). We consider only $T$ and $\mathfrak{L}_{A}$ such that $\mathfrak{L}_{A}$ has enough function symbols and $T$ includes axioms for all Skolem functions of formulas of $\mathfrak{L}_{A}$. $A$ necessary condition for $T$ to have models with sets of $L_{\infty}$-indiscernibles of any order type is that $T$ have models of arbitrarily large cardinality; this is sufficient as well.

Theorem. Let $T \subseteq \mathfrak{L}_{A}$ such that $T$ has arbitrarily large models. If $\mu$ is any order type, there is a model $\mathfrak{A}$ of $T$ such that $\mathfrak{A}$ has a set of $L_{\infty}$ indiscernibles of order type $\mu$.

Proof. We may suppose that the cardinality $|\mu|$ of $\mu$ is $\leqq \kappa$, since a set of $L_{\infty \lambda}$-indiscernibles is a set of $L_{\infty}$-indiscernibles if $\lambda \geqq \kappa$. Sup-

Received by the editors December 7, 1969.

AMS Subject Classifications. Primary 0235.

Key Words and Phrases. Indiscernibles, infinitary languages, $\eta_{\alpha}$-set. 
pose $\kappa=\boldsymbol{\aleph}_{\alpha}$; it suffices to prove that $T$ has a model $\mathfrak{A}$ with a set $X$ of $L_{\infty}$-indiscernibles of order type $\eta_{\alpha}$, since $\mu$ can be embedded in $X$ [7, pp. 334-338].

We are assuming that models of $T$ have Skolem functions for all formulas of $\mathfrak{L}_{A}$. Since $T$ has arbitrarily large models, there is a model $\mathfrak{I}$ of $T$ with a set $X$ of $\mathfrak{L}_{A}$-indiscernibles of order type $\eta_{\alpha}$ (see [6]; if $\mathfrak{L}_{A}=L_{\omega \omega}$ this is just the classical result of Ehrenfeucht-Mostowski [5]). We may suppose that $\mathfrak{U}=\mathfrak{S}(X)$, where $\mathfrak{S}(X)$ is the Skolem hull of $X$ (i.e. the submodel of $\mathfrak{A}$ whose universe $A$ is the closure of $X$ under the Skolem functions of $\mathfrak{L}_{A}$ ).

We claim that $X$ is a set of $L_{\infty x}$-indiscernibles in $\mathfrak{A}$. Let $Y \subseteq X$ be of cardinality $\left\langle\kappa=\boldsymbol{\aleph}_{\alpha}\right.$ and let $h: Y \rightarrow X$ be an order-preserving injection. Let $I$ be the set of all isomorphisms

$$
f: S \rightarrow S^{\prime}
$$

of submodels $S, S^{\prime}$ of $\mathfrak{A}$ such that $Y \subseteq S, f \mid Y=h$, and there exist $U$, $U^{\prime} \subseteq X$ such that $|U|<\kappa, S=\mathfrak{S}(U), S^{\prime}=\mathfrak{S}\left(U^{\prime}\right)$ and $f \mid U$ is an orderisomorphism of $U$ onto $U^{\prime}$. Notice that $I \neq \varnothing$ since, letting $S=\mathfrak{S}(Y)$, $S^{\prime}=\mathfrak{S}(h(Y))$, there is an extension of $h$ to an isomorphism $f: S \rightarrow S^{\prime}$. We claim that $I$ has the following property:

For any $C \subseteq A$ such that $|C|<\kappa$ and any $f \in I$, there are $f^{\prime}, f^{\prime \prime} \in I$ such that $f \subseteq f^{\prime}, f \subseteq f^{\prime \prime}, C \subseteq$ domain of $f^{\prime}$, and $C \subseteq$ range of $f^{\prime \prime}$.

It suffices to prove $\left({ }^{*}\right)$, for it follows easily by an induction on formulas of $L_{\infty x}$ that

$$
(\mathfrak{A}, y)_{y \in Y} \equiv_{\infty \kappa}(\mathfrak{A}, h y)_{y \in Y}
$$

(see Calais [2]).

So suppose $f: S \rightarrow S^{\prime}$ is in $I$ and $U, U^{\prime}$ are as in the definition of $I$. Given $C \subseteq A,|C|<\kappa$, there is a $D \subseteq X,|D|<\kappa$, such that $C \subseteq \mathfrak{S}(U \cup D)$. It is clear that in order to prove the existence of $f^{\prime}$ as required by $\left({ }^{*}\right)$, it suffices to show that we can extend $f \mid U: U \rightarrow U^{\prime}$ to an order-monomorphism: $U \cup D \rightarrow X$. We may assume $D \cap U=\varnothing$. Define an equivalence relation on $D$ by: $x \approx y$ iff $x$ and $y$ determine the same cut of $U$. Write $D=\mathrm{U}_{\sigma<\tau} D_{\sigma}$ as the union of pairwise disjoint equivalence classes $D_{\sigma}, \sigma<\tau<\kappa$. For any $\sigma<\tau$, let $U=A_{\sigma} \cup B_{\sigma}$ where $A_{\sigma}<D_{\sigma}<B_{\sigma}$. Then $f\left(A_{\sigma}\right)<f\left(B_{\sigma}\right)$ and $\left|f\left(A_{\sigma}\right)\right|<\kappa,\left|f\left(B_{\sigma}\right)\right|<\kappa$. So if

$$
E_{\sigma}=\left\{x \in X: f\left(A_{\sigma}\right)<x<f\left(B_{\sigma}\right)\right\},
$$


$E_{\sigma}$ is an $\eta_{\alpha}$-set. Therefore there exists an embedding

$$
g_{\sigma}: D_{\sigma} \rightarrow E_{\sigma} .
$$

Define $f^{\prime}: U \cup D \rightarrow X$ by: $f^{\prime}(x)=f(x)$ if $x \in U ; f^{\prime}(x)=g_{\sigma}(x)$ if $x \in D_{\sigma}$. This gives the desired extension of $f$. In a similar manner we can prove the existence of $f^{\prime \prime}$ extending $f$ with $C \subseteq$ range of $f^{\prime \prime}$. This completes the proof.

REMARKS. (1) If we assume the generalized continuum hypothesis then the proof is much simpler; for then there exists an $\eta_{\alpha}$-set $X$ of cardinality $\boldsymbol{\aleph}_{\alpha}$. Hence if $h: Y \rightarrow X$ is an order-preserving injection and $|Y|<\kappa, h$ extends to an isomorphism $h^{\prime}: X \rightarrow X$. It is immediate that

$$
(\mathfrak{A}, y)_{y \in Y}=_{\infty x}(\mathfrak{A}, h y)_{y \in Y} \text {. }
$$

(Compare the remark of Chang [3, p. 55].)

(2) Our method suffers from the same defect as that of Chang, namely the indiscernibles do not necessarily generate the model.

(3) If $\kappa=\boldsymbol{\aleph}_{\alpha}$ and $\kappa \geqq|\mu|$ the model $\mathfrak{A}$ asserted to exist in the statement of the theorem can be chosen to have cardinality $=2^{\aleph_{\beta}}$ if $\alpha=\beta+1 ; \sum_{\sigma<\alpha} 2^{\aleph_{\sigma}}$ if $\alpha$ is a limit ordinal [7].

\section{REFERENCES}

1. J. Barwise, Infinitary logic and admissible sets, J. Symbolic Logic 34 (1969), 226-252.

2. J.-P. Calais, La méthode de Fraissê dan les langages infinis, C. R. Acad. Sci. Paris 268 (1969), 785-788.

3. C. C. Chang, "Some remarks on the model theory of infinitary languages," The syntax and semantics of infinitary languages, Lecture Notes in Math., no. 72, Springer-Verlag, Berlin and New York, 1968, pp. 36-63.

4. _ - "Infinitary properties of models generated from indiscernibles," Logic, methodology and the philosophy of science. III, North-Holland, Amsterdam, 1968, pp. 9-21.

5. A. Ehrenfeucht and A. Mostowski, Models of axiomatic theories admitting automorphisms, Fund. Math. 43 (1956), 50-68. MR 18, 863.

6. J. Keisler, Model theory of $L_{\omega_{1} \omega}$, (to appear).

7. K. Kuratowski and A. Mostowski, Set theory, PWN, Warsaw and NorthHolland, Amsterdam, 1968. MR 37 \#5100.

Yale University, New Haven, Connecticut 06520 